\title{
Home respiratory polygraphy for diagnosis of sleep-disordered breathing in heart failure
}

\author{
E. Quintana-Gallego*, M. Villa-Gil ${ }^{\#}$, C. Carmona-Bernal*, G. Botebol-Benhamou", \\ Á. Martínez-Martínez ${ }^{\#}$, Á. Sánchez-Armengol*, J. Polo-Padillo ${ }^{+}$, F. Capote*
}

Home respiratory polygraphy for diagnosis of sleep-disordered breathing in heart failure. E. Quintana-Gallego, M. Villa-Gil, C. Carmona-Bernal, G. Botebol-Benhamou, Á. Martínez-Martínez, Á. Sánchez-Armengol, J. Polo-Padillo, F. Capote. (C)ERS Journals Ltd 2004.

ABSTRACT: The present study assessed the usefulness of home cardiorespiratory polygraphy as an alternative to overnight polysomnography for the diagnosis of sleepdisordered breathing in heart failure.

A total of 75 patients with chronic stable heart failure due to systolic dysfunction (left ventricular ejection fraction $\leqslant \mathbf{4 5} \%$ ) underwent polysomnography at the Virgen del Rocio Hospital and cardiorespiratory polygraphy at home. The two studies were performed in random order and within 30 days. The accuracy of polygraphical findings was determined by sensitivity and specificity obtained by area under the receiver operating characteristics (ROC) curves for apnoea-hypopnoea index (AHI) thresholds of $\geqslant 5, \geqslant 10$ and $\geqslant 15$ in the polysomnography.

The area under the ROC curve for $\mathrm{AHI} \geqslant 5, \geqslant 10$ and $\geqslant 15$ was $0.896,0.907$ and 0.862, respectively. The diagnostic accuracy of polygraphy compared with polysomnography ranged $78.6-84 \%$, with sensitivities of $68.4-82.5 \%$, and specificities of 88.6-97.8\% for the different AHI thresholds. Altogether, 29 patients had an AHI $\geqslant 10$ (central pattern 24, obstructive pattern 5). Sensitivity and specificity of home polygraphy for identifying the sleep-disordered breathing pattern was $100 \%$.

These data show that home cardiorespiratory polygraphy has a high sensitivity and specificity for the diagnosis of sleep-disordered breathing associated with heart failure. Eur Respir J 2004; 24: 443-448.
Depts of *Pneumology, ${ }^{\#}$ Cardiology, and Neurophysiology, Virgen del Rocio Hospital, and ${ }^{+}$Dept of Statistics, Sevilla University, Sevilla, Spain.

Correspondence: E. Quintana-Gallego, Dept of Pneumology, Virgen del Rocio Hospital, Manuel Siurot s/n, E-41013 Sevilla, Spain.

Fax: 34955012199

E-mail: mqg01se@saludalia.com

Keywords: Central sleep apnoea

diagnosis

heart failure

obstructive sleep apnoea

portable devices

sleep-disordered breathing

Received: December 182003

Accepted after revision: May 122004

This study was supported by a grant from Consejería de Salud, Junta de Andalucía, Spain (154/00) and by Red Respira (RTIC 03/ 11, Fondo de Investigación Sanitaria, Instituto San Carlos III).
The prevalence of heart failure has increased consistently during the last 20 yrs mainly due to an increase in the survival of patients with acute myocardial infarction, and is a major health problem in Western countries [1]. Despite recent advances in drug therapy, heart failure continues to cause high morbidity and mortality rates. It has recently been shown that sleep-related breathing disorders are common in heart failure. Approximately $40-50 \%$ of patients with stabilised left ventricular failure and adequate drug treatment may present with sleep-disordered breathing (SDB), including central sleep apnoea, commonly referred to as Cheyne-Stokes respiration $(40 \%)$ or obstructive sleep apnoea (OSA) $(11 \%)$ [2-4]. This association is particularly relevant due to the high prevalence of both conditions $[5,6]$ and the adverse prognostic implications of breathing disorders in heart failure $[7,8]$. Recent studies $[9,10]$ have provided important insight into the pathophysiology of sleep apnoea syndrome in patients with congestive heart failure, with potential therapeutic implications.

Conventional polysomnography [11] assesses the typical pattern of central sleep apnoea with Cheyne-Stokes respiration associated with heart failure, which consists of increasing-decreasing oscillations of the airflow alternating with central apnoeas followed by desaturations $[12,13]$. However, indications for sleep studies in patients with heart failure have not been definitively established [14, 15], and until other methods, such as home ambulatory monitoring [16, 17], are validated for this purpose, in-laboratory polysomnography remains the diagnostic tool of choice. The objective of this study was to determine the diagnostic accuracy of home cardiorespiratory polygraphy in the assessment of SDB in congestive heart failure and the ability to differentiate between central and obstructive respiratory events using standard polysomnography as a gold standard.

\section{Methods}

\section{Patients}

The authors studied, prospectively, patients of both sexes with stable heart failure due to systolic dysfunction (left ventricular ejection fraction (LVEF) $\leqslant 45 \%$ ) who were followed at the outpatient clinic of the Service of Cardiology, Virgen del Rocio Hospital. Patients with stable congestive heart failure, defined as those presenting no changes in their symptoms and signs or in the medication dosage during the 4 weeks prior to the study, were consecutively recruited. To be included in the study, patients were required not to have had changes in the symptoms and signs of congestive heart failure, or in drug treatment doses in the 4 weeks prior to the study. Exclusion criteria were as follows: instability of heart failure during the study, acute myocardial infarction in the previous 
3 months, unstable angina, congenital heart disease, and arterial oxygen tension $<8 \mathrm{kPa}(60 \mathrm{mmHg})$. Patients were contacted by a cardiologist to ask if they would consider taking part in the study. Subjects who agreed were evaluated to confirm their eligibility and underwent a radionuclide ventriculography to assess ventricular function. The study was approved by the Ethical Committee of the hospital and written informed consent was obtained from all participants.

All patients who fulfilled the inclusion criteria underwent a conventional overnight full-channel polysomnography in the sleep laboratory of the hospital as well as a domiciliary overnight polygraphy (unattended mode), whereby an experienced technician went to each patient's home to place the sensors and instruct the patient. The order to perform polysomnography and cardiorespiratory polygraphy was established at random, and the interval between both sleep studies was within 30 days. Analyses of recordings obtained by polysomnography and the readings of the polygraphy were performed by two independent observers who were blind to the results of the other method.

\section{Equipment}

The polysomnography consisted of continuous polygraphical recordings for a whole night from 22:00 h to 07:00 h with standardised equipment (Somnostar 4100; SensorMedics Corporation, Yorba Linda, CA, USA) with surface leads for electroencephalogram (C3/A2, C4/A1, O1/A2 and O2/A1 placements), electrooculogram, submental electromyogram, and electrocardiogram (V2). For respiratory sensors, nasal and oral signals by thermistors were used and the chest and abdominal effort was measured by two belt sensors (Healthdyne Technologies Inc., Marietta, GA, USA). Arterial oxygen saturation $\left(\mathrm{Sa}, \mathrm{O}_{2}\right)$ was recorded by digital pulse oximetry (sampling frequency of $5 \mathrm{~s}$ ) and the body position was monitored by the system. Recordings were scored manually by an experienced neurophysiologist. Polysomnographical records were scored in 30-s epochs. A complete cessation of oronasal flow $\geqslant 10 \mathrm{~s}$ was defined as apnoea. Hypopnoea was defined as $\geqslant 50 \%$ reduction in respiratory airflow lasting $\geqslant 10 \mathrm{~s}$ and accompanied by a decrease of $\geqslant 4 \%$ from baseline in $\mathrm{Sa}, \mathrm{O}_{2}$ and/or an electroencephalographical arousal [17]. An arousal was defined according to the American Sleep Disorders Association [18]. Each apnoea and hypopnoea was categorised in terms of obstructive or mixed (chest wall and abdominal movements present) and central (chest wall and abdominal movements absent). Cyclical breathing was considered when there were at least three consecutive periods of crescendo-decrescendo oscillation in airflow amplitude. Sleep data were staged according to the system of RECHTSCHAFFEN and KALES [19]. The total number of scored apnoeas and hypopneas divided by the number of hours of sleep (apnoea-hypopnoea index (AHI)) was determined for each participant. Above the AHI cut-off values, OSA was diagnosed if mixed and obstructive events represented $>29 \%$ of all events. The presence of central sleep apnoea and Cheyne-Stokes respiration (CSA-CSR) was considered when central respiratory events accounted for $\geqslant 70 \%$ of the total respiratory events in association with characteristic periodical increasing-decreasing oscillations of airflow.

The overnight home polygraphy was performed using a portable ambulatory device (Apnoescreen II; Erich Jaeger Gmbh \& CoKg, Wuerzburg, Germany) with monitoring of oronasal airflow (thermistor), chest and abdominal respiratory movements (thoracic and abdominal belts), $\mathrm{Sa}, \mathrm{O}_{2}$ (digital pulse oximeter with a sampling frequency of $5 \mathrm{~s}$ ), body position (mercury sensor), and wristband actigraphy. Analysis was carried out manually by an experienced pneumologist. Apnoea was defined as a complete cessation of airflow for $\geqslant 10 \mathrm{~s}$, hypopnoea as $\geqslant 50 \%$ reduction in respiratory airflow accompanied by a decrease of $\geqslant 4 \%$ in $\mathrm{Sa}, \mathrm{O}_{2}$ lasting $\geqslant 10 \mathrm{~s}$. Cyclical breathing was defined in the same way as that in the polysomnography. The actigraphy was used to eliminate periods of obvious movements lasting $\geqslant 1 \mathrm{~min}$. The respiratory disturbance index (RDI) was defined as the number of respiratory events (apnoeas and hypopnoeas) per $h$ of recording. CSA-CSR and OSA were defined with the same criteria as that in the polysomnography.

\section{Statistical analysis}

The diagnostic accuracy of polygraphical findings was evaluated in terms of sensitivity and specificity, obtained through the receiver operating characteristics (ROC) curves according to different AHI cut-off points in the polysomnography $(\geqslant 5, \geqslant 10$, and $\geqslant 15)$. The best cut-off point for balancing sensitivity and specificity of the test over the range of AHI thresholds was determined. To avoid inflating the data in favour of the portable device, the present study has taken into acount missing home studies in the analysis of sensitivity and specificity. Agreement between AHI (polysomnography) and RDI (respiratory polygraphy) was assessed using the method described by BLAND and ALTMAN [20]. The discriminatory ability of home polygraphy monitoring for the diagnosis of central and obstructive respiratory events was evaluated by sensitivity and specificity analyses. Interobserver agreement was estimated by kappa statistic. The level of significance was accepted at a p-value of $<0.05$.

\section{Results}

A total of 90 patients accepted to participate in the present study. Altogether, 15 subjects withdrew before finishing the protocol (one died, two underwent a cardiac transplantation, nine suffered a descompensation of their heart failure, three withdrew consent). Therefore, the study population included 75 patients, 65 males $(86.7 \%)$ and 10 females $(13.3 \%)$, with a mean \pm SD age of $56.1 \pm 11.7$ yrs. The mean LVEF was $29.3 \pm 9.5 \%$, and $85.7 \%$ of patients were in New York Heart Association functional class I-II (table 1). Seven home studies produced no interpretable data because of a total recording

Table 1.-Baseline characteristics of the study group

\section{Variable}

Age yrs

Sex M:F

Body mass index $\mathrm{kg} \cdot \mathrm{m}^{-2}$

Left ventricular ejection fraction $\%$

Causes of heart failure $\%$

Ischaemic

Idiophatic

Others

Medications \%

ACE inhibitors $\quad 100$

Diuretics

$\beta$-Blockers

100

Nitrates

38.4

Digoxin

24.7

Data as presented as mean $\pm \mathrm{SD}$, $\mathrm{n}$ or $\%$. M: male; F: female; ACE: angiotensin converting enzyme. 
Table 2.-Polysomnographical data in 75 sleep studies and cardiorespiratory polygraphical parameters in the 68 valid recordings

\begin{tabular}{|c|c|c|}
\hline Parameter & Polysomnography & Polygraphy \\
\hline Total recording time min & $395.9 \pm 30.6$ & $436.6 \pm 42$ \\
\hline Total sleep time min & $315.9 \pm 54.2$ & \\
\hline Sleep efficiency \% & $79.8 \pm 12.2$ & \\
\hline Stages $1-2$ of total sleep time $\%$ & $74.9 \pm 11.5$ & \\
\hline Stages 3-4 of total sleep time $\%$ & $12.6 \pm 8.7$ & \\
\hline Rapid-eye movement of total sleep time $\%$ & $12.1 \pm 7$ & \\
\hline AHI/RDI & $11.6 \pm 14$ & $10.5 \pm 8.7$ \\
\hline Basal $S \mathrm{a}, \mathrm{O}_{2} \%$ & $97 \pm 2.6$ & $95 \pm 1.9$ \\
\hline Nadir $\mathrm{Sa}_{\mathrm{a}} \mathrm{O}_{2} \%$ & $83.5 \pm 7.3$ & $79.7 \pm 11.6$ \\
\hline Percentage of total recoding time with $\mathrm{Sa}_{\mathrm{a}} \mathrm{O}_{2}<90 \%$ & $5.2 \pm 1$ & $5.1 \pm 2.2$ \\
\hline
\end{tabular}

Data are presented as mean \pm SD. AHI: Apnoea-hypopnoea index; RDI: respiratory disturbance index; $\mathrm{Sa}_{2} \mathrm{O}_{2}$ : arterial oxygen saturation.

time of $<3 \mathrm{~h}(\mathrm{n}=2)$, disconnection of the thermistor $(\mathrm{n}=1)$, and failure of bands $(n=4)$. There were no differences between patients with successful and unsuccessful studies regarding to age (56.3 \pm 11.9 versus $54.5 \pm 10.5)$ or $\mathrm{LVEF}(29.3 \pm 9.3$ versus $29.4 \pm 11.6)$. The time interval between both sleep studies was $13.8 \pm 8.9$ days.

Polysomnographical data of the 75 patients and polygraphical parameters of the 68 patients with valid home recordings are presented in table 2 . As previously mentioned, in the remaining analysis the lost polygraphical studies were also considered. According to polysomnographical data and using the AHI cut-off points of $\geqslant 5$, $\geqslant 10$, and $\geqslant 15$, the frequency of SDB in heart failure was $53.3 \%, 38.6 \%$ and $25.3 \%$, respectively. With the respiratory device, the diagnosis could be confirmed in $44 \%$ (33 of 75 patients), 30.6\% (23 of 75 patients) and $17.3 \%$ (13 of 75 patients).

The areas under the ROC curve of respiratory polygraphical scores for the different AHI cut-off points of the polysomnography are shown in table 3 . The best result of the area under the ROC curve $(0.907,95 \%$ CI: $0.817-0.998$, $\mathrm{p}<0.001$ ) corresponded to the AHI cut-off point of $\geqslant 10$. ROC curves for the different AHI cut-off points are depicted in figures 1-3. When considering the AHI cut-off point of $\geqslant 5$, the sensitivity and specificity obtained were $82.5 \%$ and $88.6 \%$. Accordingly, 59 of the 75 patients were correctly classified (identification or exclusion with an AHI $>5$ or $<5$ ) with the polygraphical device, with a diagnostic accuracy of $78.6 \%$. When considering an AHI cut-off point of $\geqslant 10$, the respiratory polygraphical recordings showed a sensitivity of $79.3 \%$ and a specificity of $97.8 \%$ (correct classification 63 of the 75 patients, $84 \%$ ). For an AHI cut-off point of $\geqslant 15$, the sensitivity was $68.4 \%$ and the specificity was $94.6 \%$. There were 60 patients $(80 \%)$ correctly classified with respiratory polygraphy.
Post-test sensitivity and specificity values for the best RDI cut-off points identified in the ROC curves analysis $(6.1,8.5$, 12.5) are presented in table 3.

The mean of the individual differences between polysomnographic AHI and polygraphical RDI is shown in figure 4. The mean value was $1.58 \pm 8.8$ (95\% CI: $-0.57-3.73)$.

According to the results of polysomnography, 29 patients $(38.6 \%)$ had an AHI cut-off point of $\geqslant 10$ (mean AHI: $27.8 \pm 16.5)$, with central apnoea and Cheyne-Stokes respiration in $24(82.1 \%)$ (mean AHI: 30.1 16.9$)$ and obstructive

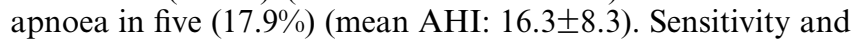
specificity of home polygraphy for detecting a central or obstructive pattern was $100 \%$, with a total concordance between the two scorers (kappa coefficient=1).

\section{Discussion}

The presented results show that home cardiorespiratory polygraphy has a high sensitivity and specificity, using conventional polysomnography as a gold standard, for diagnosis of SDB associated with heart failure, as well as for discriminating between central and obstructive apnoea. In the current patient population, the diagnostic validity of polygraphical readings compared to polysomnography was very high as shown by the areas under the ROC curves for each AHI cut-off points $(\geqslant 5, \geqslant 10$, and $\geqslant 15 ; 0.896,0.907$ and 0.862 , respectively). Concordance between AHI values and polygraphical respiratory event indexes was also high, with a mean difference of 1.58 and only five cases out of the agreement range. Moreover, in $100 \%$ of cases, home cardiorespiratory polygraphy was able to identify the pattern of SDB (central or obstructive) that was present in the polysomnography.

Table 3. - Area under the receiver operating characteristics (ROC) curve of respiratory polygraphic scores and best cut-off points for different polysomnographical diagnostic apnoea-hypopnoea indexes

\begin{tabular}{lccc}
\hline Polygraphical data & \multicolumn{2}{c}{ Polysomnography } \\
\cline { 2 - 4 } & AHI $\geqslant 5$ & AHI $\geqslant 10$ & AHI $\geqslant 15$ \\
\hline Area under the ROC curve & $0.896(0.815-0.977)$ & $0.907(0.817-0.998)$ & $0.862(0.730-0.994)$ \\
Sensitivity & $82.5(70.7-94.2)$ & $79.3(64.5-94)$ & $68.4(47.5-89)$ \\
Specificity & $88.6(74.1-97.3)$ & $97.8(93.7-100)$ & $94.6(86.1-0.99)$ \\
Cut-off point & $75(61.5-88.4)$ & 8.5 & 12.5 \\
Sensitivity & $94.3(84.4-100)$ & $93.8(69-96.5)$ & $78.9(60.6-97.2)$ \\
Specificity & & $93.5(80.7-99.3)$ & $92.9(84.4-99.5)$ \\
\hline
\end{tabular}

Data are presented as $\%(95 \%$ CI). From the ROC curve, the cut-off point that best fits the finest sensitivity/specificity profile was specifically selected. In this case, the method provided other cut-off points that balanced both sensitivity and specificity. AHI: apnoea-hypopnoea index; ROC: receiver operating characteristics. 


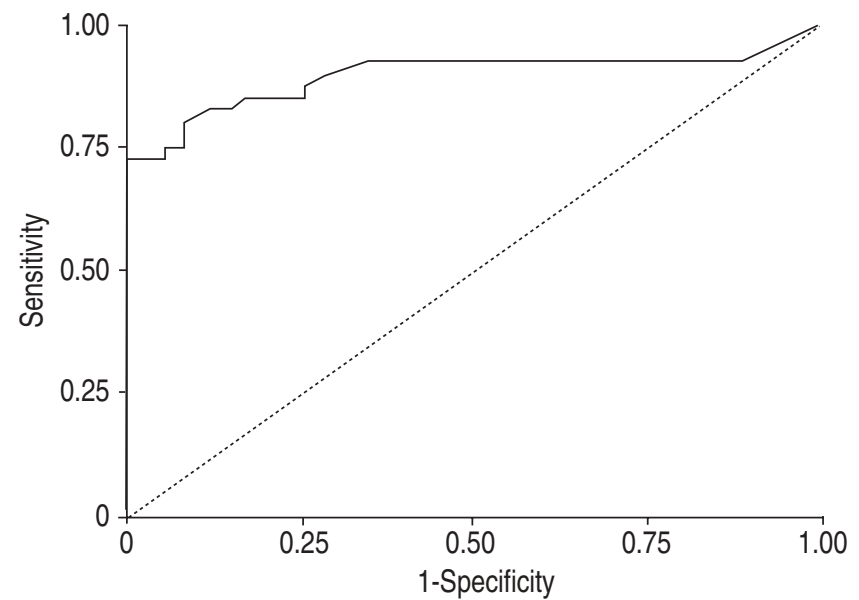

Fig. 1.- Receiver operating characteristic (ROC) referred to an apnoea-hypopnoea index $\geqslant 5$. ROC shows the sensitivity and specificity of each observed value of the respiratory disturbance index obtained from the respiratory polygraphy in relation to a full polysomnography cut-off point of five.

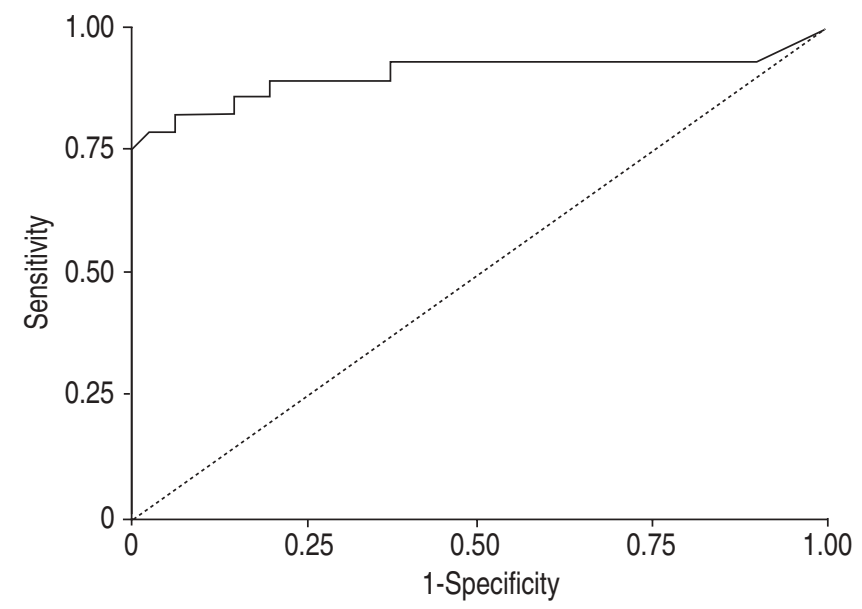

Fig. 2.-Receiver operating characteristic (ROC) referred to an apnoea-hypopnoea index $\geqslant 10$. ROC shows the sensitivity and specificity of each observed value of the respiratory disturbance index obtained from the respiratory polygraphy in relation to a full polysomnography cut-off point of 10 .

The presence of SDB in heart failure has been shown in several studies. Two previous studies $[3,4]$ have shown a frequency of central sleep apnoea associated with CheyneStokes respiration in heart failure of $33-41 \%$ for an AHI value $\geqslant 10$, which is in the range of $33 \%$ found in the current study based on a population of ambulatory patients with stable heart failure, functional class I-II for $85 \%$ of them, and prospectively studied. JAVAHERI et al. [3] studied patients attending a Veterans Hospital and were thus likely to be elderly, male and have coexistent medical problems. With regard to OSA, a prevalence of $6 \%$ in the present study was $<11 \%$ in the series of JAVAHERI et al. [3] and $31 \%$ in the series of SIN et al. [4], probably because patients with congestive heart failure and suspicion of OSA were studied. In contrast, SolIN et al. [2] studied 75 patients with chronic heart failure and the incidence of central and obstructive apnoea was $32 \%$ and $11 \%$, respectively. As in the study by Solin et al. [2], the patients in the current study were recruited consecutively

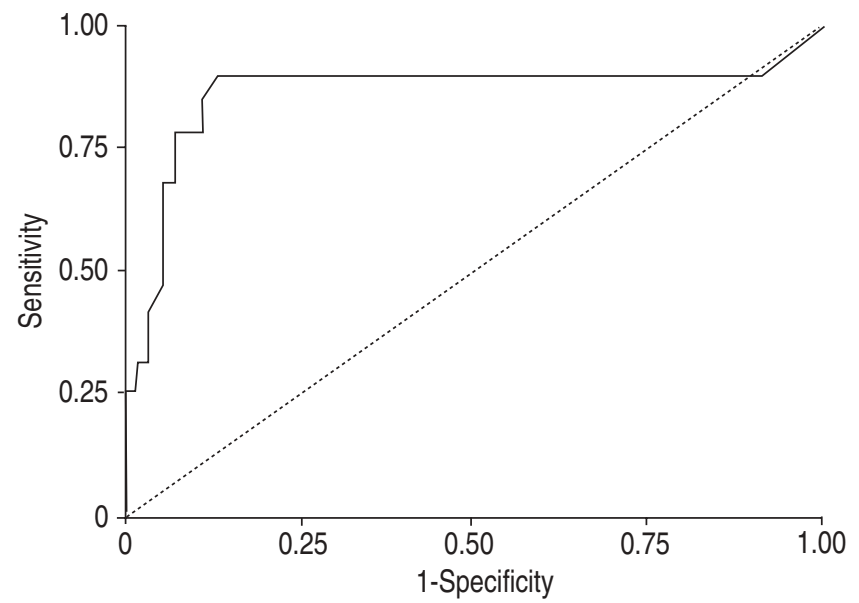

Fig. 3.-Receiver operating characteristic (ROC) referred to an apnoea-hypopnoea index $\geqslant 15$. ROC shows the sensitivity and specificity of each observed value of the respiratory disturbance index obtained from the respiratory polygraphy in relation to a full polysomnography cut-off point of 15 .

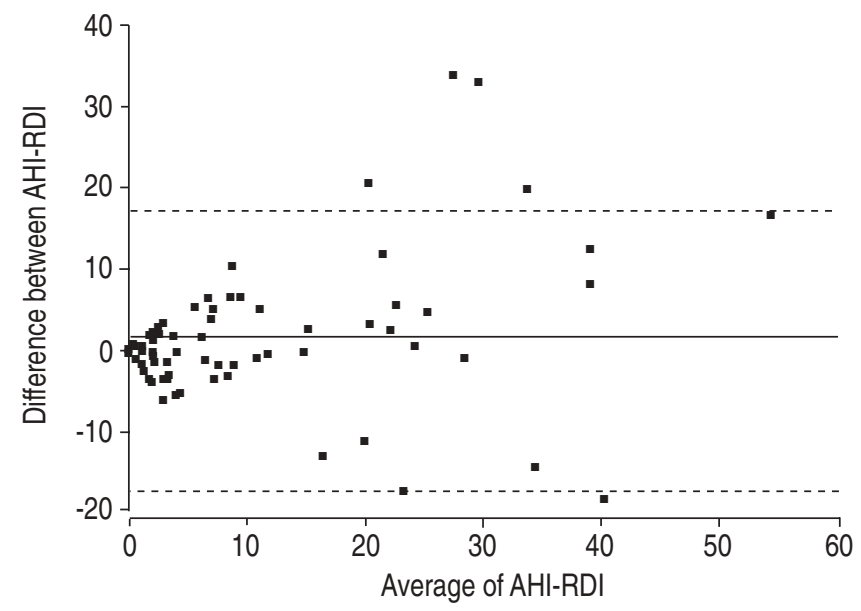

Fig. 4.-Bland-Altman plot for the respiratory disturbance index (RDI) values from respiratory polygraphy compared with the apnoea-hypopnoea index (AHI) from polysomnography. - : mean bias (1.58) of the individual differences; ---: the limits of agreement (mean bias $\pm 1.96 \mathrm{SD}$ of the differences).

from an ambulatory cardiological unit and they did not primarily complain of symptoms related with SDB.

There is a growing body of evidence that the mechanical, haemodynamic, autonomic, and chemical effects of central sleep apnoea and OSA place patients with heart failure at increased risk of accelerated disease progression [8, 21]. However, sleep studies are not routinely considered in the evaluation of patients with heart failure. Moreover, polysomnography remains the tool of choice because other home ambulatory cardiorespiratory polygraphy systems have not been validated for this purpose [21]. To date, there have been no studies assessing the accuracy of unattended cardiorespiratory polygraphy at home versus polysomnography for the diagnosis of SDB in congestive heart failure. In a previous study, STANIFORTH et al. [22] aimed to determine the prevalence of SDB within a UK heart failure population, and 41 patients underwent polysomnography to assess the validity of oximetry as a screening test for Cheyne-Stokes 
respiration. Home oximetry was a good screening test for Cheyne-Stokes respiration, with a specificity of $81 \%$ and a sensitivity of $87 \%$ for nocturnal desaturation of $\geqslant 15$ dips per h. However, the desaturation pattern did not allow differentiation between central and obstructive events.

In recent years, cardiorespiratory polygraphy has been used as an alternative to polysomnography for the diagnosis of OSA. Validation studies of different cardiorespiratory monitoring systems in the sleep laboratory setting with overnight polysomnography performed simultaneously, have shown high sensitivity and specificity values [23]. In contrast, validation studies of portable cardiorespiratory devices for domiciliary unattended sleep studies have shown variable values of sensitivity and specificity $[24,25]$. The current study was conducted to obtain relevant data on the accuracy of the home polygraphy for diagnosis purposes in a population with heart failure. In this case, the key question was whether portable monitoring at home could accurately diagnose SDB at a specific target level of pathology in patients with heart failure. Thus, a priori selected polysomnography and respiratory polygraphy thresholds of $\geqslant 5, \geqslant 10$ and $\geqslant 15$ were chosen arbitrarily because, at the present time, the definition of SDB in these patients is still unclear. Although these thresholds have been specifically selected, the ROC curves permit the obtainment of other cut-off points in the polygraphy that improve either sensitivity or specificity. In the present study, the diagnostic accuracy of home polygraphy compared with polysomnography in patients with heart failure varied between $78.9-84 \%$, with high sensitivities (between 68.4-94.6\%) and specificities (88.6-97.8\%) for all AHI cut-off points considered. These results are similar to those obtained for the diagnosis of OSA. In a study carried out with a similar methodology to that of the present study [26], ambulatory polygraphy showed a sensitivity of $73 \%$ and specificity of $80 \%$ for the diagnosis of OSA for an AHI $\geqslant 10$ in the polysomnography.

Discrepancies between polysomnography and cardiorespiratory polygraphy performed at different nights for diagnosis of SDB may be due to different factors. First, a diagnostic limitation of polygraphy is that it does not allow the accurate determination of total sleep time, so that it is necessary to use total recording time as the denominator in the calculation of RDI. As a consequence the AHI may be underestimated, with the likelihood of false negative results, especially in mild cases of SDB [23]. This probably explains that the specificity of polygraphy in the current series of patients with stable congestive heart failure was higher than the sensitivity. Secondly, there may be a temporal variability in the intensity of SDB when sleep studies are performed on different nights. In OSA, differences between two polysomnographies carried out within a short time interval are small, although there are some discrepancies in mild cases [27]. In stable congestive heart failure changes of intensity of SDB along the time have not been assessed, although it may be assumed that they may be of a greater magnitude than in OSA since the haemodynamic, symptomatic, and therapeutic profile of congestive heart failure is not static. However, the present results do not seem to support this hypothesis given that agreement between both sleep studies was high and compares favourably with that obtained in similar studies in patients with OSA. In this respect, it should be noted that patients who had a descompensation of their disease during the study were excluded, so all the subjects who underwent both sleep studies were apparently stable.

Unattended domiciliary sleep studies have some potential advantages. On the one hand, the "first night" effect is prevented since the patient does not have to sleep in an unusual environment [28] and, on the other hand, an easier and cheaper diagnostic process is achieved. However, unattended techniques have some inconveniences, such as the loss of data related to the absence of a technician during sleep recording, which would counterbalance the potential savings as sleep studies have to be repeated. In the present study series, the percentage of invalidated recordings was $9 \%$, which is similar or somewhat lower than in other studies of OSA that range 10-20\% [29]. Although it has also been discussed [30], some data suggest that unattended sleep studies at home are more favourable than an overnight polysomnography in the sleep laboratory in terms of costeffectiveness, in the diagnosis of OSA [29]. In the population of patients with heart failure, more studies are needed to determine potential savings of domiciliary sleep studies for diagnosis of SDB.

The classification of respiratory events as central or obstructive is important in the clinical context of heart failure given that the clinical significance of these patterns may be different. To quantify the respiratory effort, the most precise method is the measurement of oesophageal pressure by means of an intra-oesophageal catheter, but this is an invasive technique and difficult to perform in the daily clinical practice. In the current series, chest wall and abdominal movements were used for qualitatively estimating the respiratory effort, both in polysomnography and polygraphy, with a complete agreement in the classification of central or obstructive patterns. The present study employed thermistors to detect airflow, because other more accurate methods (such as nasal cannula) have not been used to date in unattended studies [24]. There is no agreement about criteria for defining hypopnoea in the SRBD, which occurs in patientes with HF. Although in some studies desaturation is not required [4], in others a drop in baseline $\mathrm{Sa}, \mathrm{O}_{2}$ is needed $[3,5]$. The last option was chosen because it could be safe to use corroborative data to define hypopnoeas by means of thermistor.

In summary, this is the first study that compares conventional polysomnography with unattended domiciliary cardiorespiratory polygraphy for the diagnosis of sleep-disordered breathing in patients with heart failure. In this group of patients with stable congestive heart failure, polygraphy showed a good sensitivity and a high specificity with respect to polysomnography, so that it may be a useful diagnostic tool for sleep-disordered breathing in heart failure.

Acknowledgements. The authors are indebted to M. Pulido for editing the manuscript and editorial assistance.

\section{References}

1. Braunwald E, Bristow MR. Congestive heart failure: fifty years of progress. Circulation 2000; 102: Suppl. 4, 14-23.

2. Solin P, Bergin P, Richardson M, Kaye DM, Walters EH, Naughton MT. Influence of pulmonary capillary wedge pressure on central apnea in heart failure. Circulation 1999; 99: 1574-1579.

3. Javaheri S, Parker TJ, Liming JD, et al. Sleep apnea in 81 ambulatory male patients with stable heart failure. Types and their prevalences, consequences, and presentations. Circulation 1998; 97: 2154-2159.

4. Sin DD, Fitzgerald F, Parker JD, Newton G, Floras JS, Bradley TD. Risk factors for central and obstructive sleep apnea in 450 men and women with congestive heart failure. Am J Respir Crit Care Med 1999; 160: 1101-1106.

5. Lanfranchi PA, Braghiroli A, Bosimini E, et al. Prognostic value of nocturnal Cheyne-Stokes respiration in chronic heart failure. Circulation 1999; 99: 1435-1440.

6. Ballester E, Carmona C, Egea C, Monasterio C, Izquierdo 
JL, Villasante C. Impacto del SAHS y otros trastornos respiratorios del sueño en neumología y cardiología. [Effect of sleep apnea-hypopnea syndrome and other respiratory disorders during sleep in pneumology and cardiology]. Arch Bronconeumol 2002; 38: Suppl. 3, 40-45.

7. Wilcox I, McNamara SG, Wessendorf T, Willson GN, Piper AJ, Sullivan CE. Prognosis and sleep disordered breathing in heart failure. Thorax 1998; 53: Suppl. 3, 33-36.

8. Hanly PJ, Zuberi-Khokhar NS. Increased mortality associated with Cheyne-Stokes respiration in patients with congestive heart failure. Am J Respir Crit Care Med 1996; 153: 272-276.

9. Yan AT, Bradley TD, Liu PP. The role of continuous positive airway pressure in the treatment of congestive heart failure. Chest 2001; 120: 1675-1685.

10. Kaneko Y, Floras JS, Usui K, et al. Cardiovascular effects of continuous positive airway pressure in patients with heart failure and obstructive sleep apnea. N Engl J Med 2003; 348: 1233-1241.

11. The Report of an American Academy of Sleep Medicine Task Force. Sleep-related breathing disorders in adults: recommendations for syndrome definition and measurement techniques in clinical research. Sleep 1999; 22: 667-689.

12. Naughton MT. Pathophysiology and treatment of CheyneStokes respiration. Thorax 1998; 53: 514-518.

13. Trinder J, Merson R, Rosenberg JI, Fitzgerald F, Kleiman J, Douglas Bradley T. Pathophysiological interactions of ventilation, arousals, and blood pressure oscillations during Cheyne-Stokes respiration in patients with heart failure. $\mathrm{Am}$ J Respir Crit Care Med 2000; 162: 808-813.

14. Leung RS, Bradley TD. Sleep apnea and cardiovascular disease. Am J Respir Crit Care Med 2001; 164: 2147-2165.

15. Köhnlein T, Welte T, Tan LB, Elliott MW. Central sleep apnoea syndrome in patients with chronic heart disease: a critical review of the current literature. Thorax 2002; 57: 547554.

16. Polysomnography Task Force, American Sleep Disorders Association Standards of Practice Committee. Practice parameters for the indications for polysomnography and related procedures. Sleep 1997; 20: 406-422.

17. Barbé F, Amilibia J, Capote F, et al. Diagnóstico del síndrome de apneas obstructivas durante el sueño. Informe de consenso del Área de Insuficiencia Respiratoria y Trastornos del Sueño. [Diagnosis of obstructive sleep apnoea syndrome. Consensus report from the Respiratory
Insufficiency and Sleep Disorders Group]. Arch Bronconeumol 1995; 31: 460-462.

18. American Sleep Disorders Association. EEG arousals: scoring rules and examples: a preliminary report from the Sleep Disorders Atlas Task Force of the American Sleep Disorders Association. Sleep 1992; 15: 173-184.

19. Reschtschaffen A, Kales A. A manual of standardized terminology, techniques and scoring system for sleep stages of human subjects. Washington DC, US Government Printing Office, 1979; National Institutes of Health Publication No. 204

20. Bland JM, Altman DG. Statistical methods for assessing agreement between two methods of clinical measurement. Lancet 1986; 1: 307-310.

21. Bradley DT, Floras JS. Sleep apnea and heart failure. Part II: central sleep apnea. Circulation 2003; 107: 1822-1826.

22. Staniforth AD, Kinnear WJ, Starling R, Cowley AJ. Nocturnal desaturation in patients with stable heart failure. Heart 1998; 79: 394-399.

23. Douglas NJ, Thomas S, Jan MA. Clinical value of polysomnography. Lancet 1992; 339: 347-350.

24. Flemons WW, Littner MR, Rowley JA, et al. Home diagnosis of sleep apnea: A systematic review of the literature. Chest 2003; 124: 1543-1579.

25. Cheeson AL, Berry RB, Pack A. Practice parameters for the use of portable monitoring devices in the investigation of suspected obstructive sleep apnea in adults. Sleep 2003; 26 : 907-913.

26. Parra O, Garcia-Esclasans N, Montserrat JM, et al. Should patients with sleep apnoea/hypopnoea syndrome be diagnosed and managed on the basis of home sleep studies? Eur Respir J 1997; 10: 1720-1724.

27. Meyer TJ, Eveloff SE, Kline LR, Millman RP. One negative polysomnogram does not exclude obstructive sleep apnea. Chest 1993; 103: 756-760.

28. Stiller RA, Strollo PJ, Sanders MH. Unattended recording in the diagnosis and treatment of sleep-disordered breathing. Unproven accuracy, untested assumptions, and unready for routine use. Chest 1994; 105: 1306-1309.

29. Golpe R, Jiménez A, Carpizo R. Home sleep studies in the assessment of sleep apnea/hypopnea syndrome. Chest 2002; 122: $1156-1161$.

30. Chervin RD, Murman DL, Malow BA, Totten V. Costutility of three approaches to the diagnosis of sleep apnea: polysomnography, home testing, and empirical therapy. Ann Intern Med 1999; 130: 496-505. 